\title{
The role of uncoupling protein 3 in fatty acid metabolism: protection against lipotoxicity?
}

\author{
Patrick Schrauwen* and Matthijs K. C. Hesselink \\ Nutrition and Toxicology Research Institute Maastricht (NUTRIM), Departments of Human Biology and \\ Movement Sciences, Maastricht University, PO Box 616, 6200 MD Maastricht, The Netherlands
}

\begin{abstract}
The physiological function of the mitochondrial uncoupling protein (UCP), UCP3, is still under debate. There is, however, ample evidence to indicate that, in contrast to UCP1, the primary function of UCP3 is not the dissipation of energy. Rather, several lines of evidence suggest that UCP3 is associated with cellular fatty acid metabolism. The highest levels of expression of UCP3 have been found in type 2 glycolytic muscle fibres, and fasting and high-fat diets up regulate UCP3. This up-regulation is most pronounced in muscle with a low fat oxidative capacity. Acute exercise also up regulates UCP3, and this effect has been shown to be a result of the exercise-induced increase in plasma fatty acid levels. In contrast, regular physical activity, which increases fat oxidative capacity, reduces UCP3 content. Based on these data it has been postulated that UCP3 functions to export those fatty acids that cannot be oxidized from the mitochondrial matrix, in order to prevent fatty acid accumulation inside the matrix. Several experiments have been conducted to test this hypothesis. Blocking carnitine palmitoyltransferase 1, thereby reducing fat oxidative capacity, rapidly induces UCP3. High-fat diets, which increase the mitochondrial supply of fatty acids, also up regulate UCP. However, feeding a similar amount of medium-chain fatty acids, which can be oxidized inside the mitochondrial matrix and therefore do not need to be exported from the matrix, does not affect UCP3 protein levels. In addition, UCP3 is increased in patients with defective $\beta$-oxidation and is reduced after restoring oxidative capacity. In conclusion, it is suggested that UCP3 has an important physiological function in facilitating outward transport from the mitochondrial matrix of fatty acid anions that cannot be oxidized, thereby protecting against lipid-induced mitochondrial damage.
\end{abstract}

Uncoupling protein 3: Lipotoxicity: Fatty acid metabolism

The coupling between oxidative respiration, resulting in a proton gradient across the inner mitochondrial membrane, and ATP production is not $100 \%$ efficient. Isolated mitochondria show respiration in the absence of ADP, indicating that protons are transferred across the inner mitochondrial membrane without being coupled to ADP phosphorylation. This process, termed mitochondrial uncoupling, has been suggested to result in heat production and a reduction in energy efficiency. In brown adipose tissue (BAT) the mitochondrial uncoupling protein (UCP), $\mathrm{UCP} 1$, has the specific function of uncoupling oxidative respiration from ATP production, thereby producing heat for the regulation of body temperature. In man, however, the scarcity of BAT has motivated researchers to search for homologues of BAT-specific UCP1. In 1997 the discovery of UCP2 (Fleury et al. 1997) and UCP3 (Boss et al. 1997) was therefore received with great enthusiasm, especially since these novel UCP were also shown to be able to uncouple mitochondrial respiration in vitro. UCP3 is specifically expressed in skeletal muscle in man and it was suggested that UCP3 could contribute to skeletal muscle energy metabolism. Indeed, several associations between the UCP3 gene and its product and energy metabolism have been found (for recent review, see Schrauwen \& Hesselink, 2003).

Although at present there is ample evidence that UCP3 is able to uncouple mitochondria, and that there are associations between UCP3 and energy metabolism, the prevailing opinion is that the primary function of UCP3 is not the regulation of energy metabolism. This conclusion is based on, among other factors: (1) the finding that UCP3 knock-out mice have no apparent phenotype, and have a

\footnotetext{
Abbreviations: BAT, brown adipose tissue; CPT, carnitine palmitoyltransferase; UCP, uncoupling protein. *Corresponding author: Dr P. Schrauwen, fax +31 43 3670976, email p.schrauwen@hb.unimaas.nl
} 
normal metabolic rate even though their mitochondria are more tightly coupled (Gong et al. 2000); (2) UCP3 mRNA expression is up regulated in the fasting state, when energy conservation is expected (Millet et al. 1997). A recent study (Hesselink et al. 2003) has shown that a diet-induced up-regulation of UCP3 does not affect mitochondrial uncoupling. In healthy lean subjects consuming isoenergetic high-fat $v$. low-fat diets $(60 \%$ of energy as fat $v .30 \%$ of energy as fat, with identical protein levels) there was a significant $(P<0.05)$ increase in UCP3 protein levels within the physiological range within $7 \mathrm{~d}$. Following severe exhaustive ischaemic contractile activity mitochondrial coupling (determined in vivo by measuring creatine phosphate resynthesis rate after blood flow had been reinstated) is not affected, despite a $44 \%$ increase in UCP3 protein content and the presence of all potential physiological modulators of UCP3 activity (Hesselink et al. 2003). These findings suggest that mitochondrial uncoupling may not be the principal function of UCP3 in vivo in man.

In particular, the finding that UCP3 mRNA expression is up regulated during fasting (Millet et al. 1997) led researchers to investigate a potential role for UCP3 in fatty acid metabolism. In the remainder of the present review a potential role for UCP3 in fatty acid metabolism will be discussed.

\section{Fatty acids, high-fat diets and uncoupling protein 3}

As mentioned previously, an early finding in human subjects was that fasting up regulates UCP3 mRNA expression (Millet et al. 1997). This finding has been confirmed in rodents, in which it has also been shown that refeeding rapidly down regulates UCP3 mRNA expression (Samec et al. 1998). In the fasted state plasma NEFA levels are elevated, but they are rapidly reduced on refeeding. Since NEFA are potent ligands for PPAR, which are known to be involved in the regulation of genes associated with lipid metabolism, it has been suggested that the up-regulation of UCP3 with fasting could be explained by increased NEFA levels. Indeed, it has been shown that the effect of fasting on UCP3 mRNA expression can be explained by plasma NEFA levels (Weigle et al. 1998).

In addition to fasting, high-fat feeding has also been shown to up regulate UCP3 mRNA expression (Samec et al. 1998). As mentioned earlier, high-fat diet-induced up-regulation of UCP3 protein in human subjects has been confirmed recently (Hesselink et al. 2003). In this context, it is interesting to note that, at least in human subjects, consumption of high-fat diets does not necessarily result in increased plasma NEFA levels, suggesting that under these conditions NEFA are not necessarily responsible for induction of UCP3 in skeletal muscle.

\section{Muscle fibre type-specific expression of uncoupling protein 3}

Interestingly, several studies have revealed that the effect of fasting and high-fat feeding on UCP3 is dependent on the type of muscle under study. Thus, it has been shown that the effects of starvation and refeeding on UCP3
mRNA expression are more pronounced in fast glycolytic (white) muscles than in slow oxidative (red) muscles (Samec et al. 1998). These results have been interpreted as evidence for a role of UCP3 in lipid utilization, since fast glycolytic muscles have a greater capacity to shift between glucose and lipid as substrates under starved or refed conditions. In accordance with this reasoning, it has been found in human subjects that consumption of high-fat diets for 4 weeks increases UCP3 mRNA expression and that this high-fat diet-induced up-regulation of UCP3 mRNA is most pronounced in subjects with a high percentage of fast glycolytic muscle fibres (Schrauwen et al. 2001b). As these results suggest that UCP3 might be differentially expressed in glycolytic and oxidative muscle fibres, UCP3 protein expression in type 1 (oxidative), type $2 \mathrm{a}$ (glycolytic) and type 2b (fast glycolytic) muscle fibres was determined directly using immunofluorescence. It was found that UCP3 has the highest level of expression in type $2 \mathrm{~b}$ muscle fibres and the lowest level of expression in type 1 muscle fibres (Hesselink et al. 2001). This finding has been confirmed and quantified recently by Russell et al. $(2003 b)$.

\section{Acute exercise, endurance training and uncoupling protein 3}

In relation to exercise it is important to distinguish the effects of acute exercise from the effects of exercise training. Most studies have reported that acute exercise up regulates UCP3 at the mRNA level. A period of $2 \mathrm{~h}$ of treadmill running induces a $252 \%$ increase in UCP3 mRNA in white gastrocnemius and a $63 \%$ increase in red gastrocnemius (Cortright et al. 1999). In human subjects $4 \mathrm{~h}$ of cycling exercise induces an increase in transcriptional activity of UCP3 of approximately $600 \%$ (Pilegaard et al. 2000).

Although these results can be interpreted as evidence that exercise per se up regulates UCP3, it is also well known that acute exercise in the fasted state is accompanied by high levels of plasma NEFA, which could potentially explain the induction of UCP3 mRNA. In this context, a comparison of the effect of acute exercise on UCP3 mRNA expression in the fasted and glucose-fed state (Schrauwen et al. 2002a) has shown that there are marked differences in plasma NEFA levels during and after exercise. UCP3 mRNA is up regulated $4 \mathrm{~h}$ after exercise in the fasted state, but glucose feeding completely abolishes this effect, suggesting that the elevation of plasma NEFA levels is responsible for the exercise-induced upregulation of UCP3 mRNA. This explanation is consistent with the finding of Tsuboyama-Kasaoka et al. (1998) that there is a $4 \cdot 7$-fold up-regulation of UCP 3 mRNA $3 \mathrm{~h}$ after exercise but a down-regulation when measured $44 \mathrm{~h}$ after exercise.

In contrast to acute exercise, regular endurance training results in reduced UCP3 mRNA and protein levels. Thus, when rats are subjected to an endurance training programme of stepwise incremental loads for 4 weeks followed by another 4 weeks at the same level of training volume and intensity, UCP3 is significantly down regulated in soleus and anterior tibialis muscle, with the most prominent 
decrease in the anterior tibialis (less oxidative) muscle (Boss et al. 1998). In endurance-trained human subjects also UCP3 protein levels are significantly lower than those of physically fit but untrained controls (for soleus muscle $P<0.03$ and for anterior tibialis muscle $P<0.005$; Russell et al. 2003b). In addition, middle-aged sedentary subjects who have undertaken a mild activity programme for 3 months (three times per week at $40 \%$ maximal power output for approximately $2 \mathrm{~h} /$ week) show a decrease in the UCP3 protein content of approximately 30\% (Schrauwen et al. 2001c). It has been shown very recently that a reduction in UCP3 protein content can already be detected after a 2-week supervised endurance-training programme (Schrauwen \& Hesselink, 2003). Interestingly, in these and other studies it has been found that the level of $\mathrm{UCP} 3$ protein is related to measures of energy efficiency, suggesting that the down-regulation of UCP3 by endurance training might be beneficial for endurance performance.

\section{Physiological function of uncoupling protein 3: mitochondrial export of fatty acid anions?}

Based on the data presented earlier, as well as other findings, there is evidence that the physiological function of UCP3 is related to fatty acid metabolism. Important in this line of thinking is the observation that the uncoupling activity of UCP3 can be a result of the ability to transport fatty acid anions across the inner mitochondrial membrane out of the mitochondrial matrix (Skulachev, 1999). Thus, UCP3 could actively participate in fatty acid anion transport. What could be the physiological reason for such fatty acid anion export? When the regulation pattern of UCP3 is studied, it is clear that UCP3 is up regulated when there is an abundant fatty acid supply to the mitochondria, such as during fasting, high NEFA levels, acute exercise and high-fat feeding (Schrauwen et al. 2001c). Importantly, the up-regulation of UCP3 under these conditions is most pronounced in type 2 (glycolytic) muscle fibres, which have the lowest capacity to oxidize fatty acids. On the other hand, down-regulation of UCP3 occurs when fatty acid oxidation is increased or plasma NEFA levels are lowered, such as with endurance training and weight reduction (Schrauwen et al. 2001c). Also, UCP3 levels are low in type 1 muscle fibres, which have a high fat oxidative capacity. In conditions in which there is a mismatch between the supply of fatty acids and the oxidative capacity of the mitochondria, fatty acids that cannot be oxidized will accumulate in the sarcoplasm. As the outer mitochondrial membrane is permeable to fatty acids, these fatty acids can reach the inner mitochondrial membrane, where they can partition into the phospholipid bilayer and flip-flop to the matrix side of the inner mitochondrial membrane and then be released into the mitochondrial matrix. Since there is a proton gradient across the inner mitochondrial membrane (built up by oxidative phosphorylation and used to phosphorylate ADP to ATP), fatty acids that enter the mitochondrial matrix will be deprotonated and a fatty acid anion formed. Since fatty acid anions cannot 'flip-flop' back and cannot be oxidized inside the matrix, these fatty acid anions are trapped inside the mitochondrial matrix. Since UCP3 can transport fatty acid anions, it has been proposed that the physiological role of UCP3 might be to export fatty acid anions from the matrix, thereby preventing the accumulation of fatty acid anions inside the matrix (Schrauwen et al. 2001c). It should be noted that by doing so UCP3 also lowers the proton gradient and thus acts as a UCP. However, in this hypothesis the uncoupling activity of UCP3 is a side effect of its primary function, which is to protect mitochondria against accumulation of fatty acids (lipotoxicity). Recently, a slight variation of this hypothesis has been postulated, in which UCP3 does not export fatty acid anions, but fatty acid peroxides (Goglia \& Skulachev, 2003). It is important to note that this suggestion is in line with the 'lipotoxicity' hypothesis of Schrauwen et al. (2001c), and that in both hypotheses UCP3 protects the mitochondria against the deleterious effect of fatty acids inside the matrix.

\section{Evidence for the function of uncoupling protein 3 as an exporter of fatty acid anions?}

Although the proposed function of UCP3 as a fatty acid anion exporter fits well with most of the available data in the literature in which UCP3 was determined, these studies were not designed to test the function of UCP3. In order to do so, several studies have been performed in order to provide more direct evidence for the 'lipotoxicity' hypothesis. First, the capacity of Etomoxir to inhibit carnitine palmitoyltransferase (CPT) 1 was utilized. In order to facilitate mitochondrial oxidation fatty acids need to be activated to fatty acyl-CoA inside the sarcoplasm. However, in order to pass the inner mitochondrial membrane fatty acyl-CoA needs to be converted to fatty acylcarnitine, the conversion being catalysed by CPT1. In the matrix fatty acylcarnitine is reconverted to fatty acyl-CoA by CPT2 and this fatty acyl-CoA can subsequently enter the $\beta$-oxidation and tricarboxylic acid cycles. It is well known that in this process the activity of CPT1 is rate-limiting. Thus, blocking CPT1 will largely reduce the fat oxidative capacity of mitochondria, with the consequence that fatty acids will accumulate in the sarcoplasm. Then, according to the 'lipotoxicity' hypothesis described earlier these fatty acids may enter the mitochondrial matrix, and UCP3 is needed to protect the mitochondria against mitochondrial fatty acid accumulation. Thus, Etomoxir and a placebo were given to human subjects for $36 \mathrm{~h}$, while they consumed high-fat diets (to increase fatty acid supply), and fat oxidation was measured using respiration chambers. After $36 \mathrm{~h}$ UCP3 protein content was measured in biopsies taken after the subjects left the respiration chamber. It was found that $24 \mathrm{~h}$ fat oxidation is indeed inhibited, by approximately $15-20 \%$, and in accordance with the 'lipotoxicity' hypothesis UCP3 protein content is rapidly induced, by $67 \%$ (Schrauwen et al. 2002b). Furthermore, there is a negative correlation between the decrease in fat oxidation after Etomoxir treatment and the increase in UCP3 protein. It should be noted that $36 \mathrm{~h}$ is a very short period of time to allow up-regulation of mitochondrial proteins, further illustrating the impact of this finding. There has recently been confirmation of these findings in rats; Etomoxir treatment for $8 \mathrm{~d}$ resulted in an approximately 2 -fold decrease 
in CPT1 activity and an approximately 2-fold increase in UCP3 (Schrauwen et al. 2003).

The up-regulation of UCP3 by high-fat diets, as mentioned earlier, is also compatible with the 'lipotoxicity' hypothesis that UCP3 acts as a fatty acid anion (or fatty acid peroxides) exporter. On a high-fat diet the continuous high supply of fatty acids to the mitochondria will exceed the fatty acid oxidative capacity, and fatty acids will accumulate in the sarcoplasm. These fatty acids could enter the mitochondrial matrix and UCP3 would again be needed to protect the mitochondria against these fatty acids, explaining the induction of UCP3 by high-fat diets. However, this line of reasoning does not hold for all fatty acids. In contrast to long-chain fatty acids, which need to be activated to fatty acyl-CoA inside the sarcoplasm to allow mitochondrial oxidation, medium-chain fatty acids can also be activated inside the mitochondrial matrix and do not need CPT1 for mitochondrial uptake. Thus, even when medium-chain fatty acids accumulate in the sarcoplasma, and subsequently reach the mitochondrial matrix, they can still be activated to fatty acyl-CoA and be oxidized. Obviously, these fatty acids do not need to (and should not) be exported from the matrix. Thus, if UCP3 does export from the matrix those fatty acids that cannot be oxidized, it should not be induced with a high-fat diet consisting of mainly medium-chain triacylglycerols. Consequently, rats were fed for $14 \mathrm{~d}$ on high-fat diets comprising only either long-chain fatty acids (46\% energy as fat, of which $79 \mathrm{~g} / 100 \mathrm{~g}$ was $\mathrm{C}_{16}$ and $20 \mathrm{~g} / 100 \mathrm{~g}$ was $\mathrm{C}_{18}$ ) or medium-chain fatty acids $(46 \%$ energy as fat, of which $60 \mathrm{~g} / 100 \mathrm{~g}$ was $\mathrm{C}_{8}$ and $40 \mathrm{~g} / 100 \mathrm{~g}$ was $\mathrm{C}_{10}$ ), and the gastrocnemius muscle UCP3 protein levels measured. In accordance with earlier reports the UCP3 protein levels in the high-fat long-chain triacylglycerol group showed a 2 -fold increase. In contrast, $14 \mathrm{~d}$ of high-fat medium-chain triacylglycerols feeding did not affect UCP3 protein levels (Schrauwen et al. 2003). It is important to note that this differential response to a diet comprising long-chain fatty acids $v$. medium-chain fatty acids was accompanied by a similar rise in plasma NEFA levels in these rats. Again, this study indicates that increased fatty acid availability only affects UCP3 content if the fatty acid load to the mitochondria exceeds the mitochondrial fat oxidative capacity.

According to the 'lipotoxicity' hypothesis a normal physiological response to a low fat oxidative capacity would be an increased UCP3 protein content. As mentioned earlier, inhibition of CPT1, leading to decreased fat oxidation, does indeed increase UCP3 protein levels (Schrauwen et al. 2002b). To examine whether this physiological response is also present under pathological conditions that hinder fat oxidative capacity, UCP3 protein levels have been determined in patients suffering from riboflavin-responsive multiple acyl-CoA dehydrogenase deficiency. Patients with untreated riboflavin-responsive multiple acyl-CoA dehydrogenase deficiency show decreased oxidative capacity for short $\left(\mathrm{C}_{2}\right)$-, medium $\left(\mathrm{C}_{8}\right)$ and long $\left(\mathrm{C}_{16}\right)$-chain fatty acids, caused by reduced activity of flavin-dependent acyl-CoA dehydrogenases (Russell et al. 2003a). Riboflavin-responsive multiple acyl-CoA dehydrogenase deficiency can be alleviated by treatment with riboflavin, which restores fat oxidative capacity to control values. In accordance with the 'lipotoxicity' hypothesis, UCP3 protein levels are indeed increased 1.7-fold in patients with riboflavin-responsive multiple acyl-CoA dehydrogenase deficiency. Riboflavin treatment restores fatty acid oxidative capacity and reduces UCP3 protein levels to control values (Russell et al. 2003a). These data show that, in a longitudinal design, restoration of fat oxidative capacity with simultaneous decline in fatty acid supply is followed by a rapid decline in UCP3 protein. Once more, this study supports the 'lipotoxicity' hypothesis that UCP3 exports fatty acid anions (or fatty acid peroxides) from the mitochondrial matrix.

\section{Physiological relevance of uncoupling protein 3 as an exporter of fatty acid anions?}

Diabetes is a condition that is clearly characterized by defects in fatty acid metabolism (Kelley \& Mandarino, 2000). In the long term, elevated plasma fatty acid levels as seen in diabetes will lead to the accumulation of fatty acids and triacylglycerols in non-adipose tissues such as the $\beta$-cell, skeletal muscle and heart. In relation to the heart it is generally assumed that lipotoxicity, defined as the negative effects of an excess of lipids, contributes to the development of diabetic cardiomyopathy. In this context it has been shown that rats rendered diabetic by treatment with streptozotocin do indeed show decreased fatty acid oxidative capacity and increased UCP3 mRNA levels in the heart (Hidaka et al. 1999). This finding has recently been confirmed at the UCP3 protein level; UCP3 protein levels in heart muscle of rats treated with streptozotocin for 4 weeks were found to be several fold higher than those of control rats. Interestingly, this increase in UCP3 is closely associated with increased levels of sarcoplasmic fatty acids (DM Hasselbaink, P Schrauwen, MKC Hesselink, $M$ van Bilsen and GJ van der Vusse, unpublished results). This observation directly links the change in UCP3 to the change in sarcoplasmic fatty acids in the non-esterified state, and further provides evidence that UCP3 protects mitochondria against excess amounts of fatty acids and/or lipotoxicity.

In this context it is important to note that it has indeed been shown that lipotoxicity is a result of a cellular accumulation of fatty acids and not triacylglycerols (Listenberger et al. 2003). Evidence for this physiological relevance of UCP3 comes from a study of Brand et al. (2002), who showed that mice lacking UCP3 are characterized by increased lipid peroxidation and increased mitochondrial damage. Consequently, lack of UCP3 could lead to disturbances in fatty acid oxidation, explaining the reported aberrations in fat oxidation in human subjects with the exon 6 splice donor mutation (Argyropoulos et al. 1998) and in UCP3 knock-out mice (Bezaire et al. 2001). Intriguingly, patients with type 2 diabetes are characterized by a $50 \%$ reduction in UCP3 protein levels (Schrauwen et al. 2001a), have low fat oxidative capacity and have been found to be more susceptible to mitochondrial DNA damage in skeletal muscle (Liang et al. 1997). Moreover, it has very recently been found that skeletal muscle of type 2 diabetic subjects is characterized by smaller and 
damaged mitochondria (Kelley et al. 2002). It is tempting to speculate that defects in the regulation of UCP3 in type 2 diabetes contribute to these morphological changes. In this context, it has recently been found that a lifestyle intervention, consisting of dietary advice and a physical activity programme, for a period of 1 year up regulates UCP3 protein in subjects with an impaired glucose tolerance (M Mensink, P Schrauwen, MKC Hesselink, E Kornips, G Schaart, WHM Saris and EE Blaak, unpublished results).

\section{Conclusion and future directions}

Currently, 7 years after its discovery, there is compelling evidence to indicate that the primary function of UCP3 is not associated with the regulation of energy metabolism. The evidence that the physiological function of UCP3 is associated with fatty acid metabolism has been reviewed. More specifically, evidence has been provided for the hypothesis that UCP3 is a transporter of fatty acid anions (or fatty acid peroxides as recently suggested by Goglia \& Skulachev, 2003) out of the mitochondrial matrix. By doing so, UCP3 could prevent accumulation of fatty acid anions (or peroxides) inside the matrix and protect mitochondria against the detrimental effects of high fatty acid levels. Clearly, future studies are needed to test this concept using, among other approaches, rodent models that under- or overexpress UCP3. In addition, studies are needed to test the physiological relevance of UCP3. It is recognized that lipotoxicity might have a major impact on mitochondrial functioning, and a role for UCP3 in the protection against lipotoxicity therefore deserves further study. It would be particularly interesting to study the role of lipotoxicity in the metabolic disturbances observed in type 2 diabetes, and whether the low UCP3 content that is observed in type 2 diabetes could contribute to lipidinduced mitochondrial dysfunction.

\section{Acknowledgement}

The authors would like to thank Professor G. J. van der Vusse, Dr A. Russell, Dr M. Mensink and J. Hoeks, for allowing them to refer to their encouraging data, which was awaiting publication when the present paper was being prepared. The research of P.S. has been made possible by fellowships from the Royal Netherlands Academy of Arts and Sciences and the Netherlands Organization for Scientific Research (NWO).

\section{References}

Argyropoulos G, Brown AM, Willi SM, Zhu J, He Y, Reitman M, Geveo SM, Spruill I \& Garvey WT (1998) Effects of mutations in the human uncoupling protein 3 gene on the respiratory quotient and fat oxidation in severe obesity and type 2 diabetes. Journal of Clinical Investigation 102, $1345-1351$.

Bezaire V, Hofmann W, Kramer JK, Kozak LP \& Harper ME (2001) Effects of fasting on muscle mitochondrial energetics and fatty acid metabolism in Ucp3(-/-) and wild-type mice. American Journal of Physiology 281, E975-E982.
Boss O, Samec S, Desplanches D, Mayet M-H, Seydoux J, Muzzin P \& Giacobino J-P (1998) Effect of endurance training on mRNA expression of uncoupling proteins 1,2 and 3 in the rat. FASEB Journal 12, 335-339.

Boss O, Samec S, Paoloni-Giacobino A, Rossier C, Dulloo A, Seydoux J, Muzzin P \& Giacobino J-P (1997) Uncoupling protein-3: a new member of the mitochondrial carrier family with tissue-specific expression. FEBS Letters 408, 39-42.

Brand MD, Pamplona R, Portero-Otin M, Requena JR, Roebuck SJ, Buckingham JA, Clapham JC \& Cadenas S (2002) Oxidative damage and phospholipid fatty acyl composition in skeletal muscle mitochondria from mice underexpressing or overexpressing uncoupling protein 3. Biochemical Journal 368, 597-603.

Cortright RN, Zheng D, Jones JP, Fluckey JD, DiCarlo SE, Grujic D, Lowell BB \& Dohm GL (1999) Regulation of skeletal muscle UCP-2 and UCP-3 gene expression by exercise and denervation. American Journal of Physiology 276, E217-E221.

Fleury C, Neverova M, Collins S, Raimbault S, Champigny O, Levi-Meyrueis C, Bouillaud F, Seldin MF, Surwit RS, Ricquier D \& Warden CH (1997) Uncoupling protein-2: a novel gene linked to obesity and hyperinsulinemia. Nature Genetics $\mathbf{1 5}$, 269-273.

Goglia F \& Skulachev VP (2003) A function for novel uncoupling proteins: antioxidant defense of mitochondrial matrix by translocating fatty acid peroxides from the inner to the outer membrane leaflet. FASEB Journal 17, 1585-1591.

Gong DW, Monemdjou S, Gavrilova O, Leon LR, MarcusSamuels B, Chou CJ, Everett C, Kozak LP, Li C, Deng C, Harper ME \& Reitman ML (2000) Lack of obesity and normal response to fasting and thyroid hormone in mice lacking uncoupling protein-3. Journal of Biological Chemistry 275, 16251-16257.

Hesselink MKC, Greenhaff PL, Constantin-Teodosiu D, Hultman E, Saris WHM, Nieuwlaat R, Schaart G, Kornips E \& Schrauwen P (2003) Increased uncoupling protein 3 content does not affect mitochondrial function in human skeletal muscle in vivo. Journal of Clinical Investigation 111, 479-486.

Hesselink MKC, Keizer HA, Borghouts LB, Schaart G, Kornips CFP, Slieker LJ, Sloop KW, Saris WHM \& Schrauwen P (2001) Protein expression of UCP3 differs between human type 1, type $2 \mathrm{a}$ and type 2b fibers. FASEB Journal 15, 10.1096/ fj.1000-0517fje.

Hidaka S, Kakuma T, Yoshimatsu H, Sakino H, Fukuchi S \& Sakata T (1999) Streptozotocin treatment upregulates uncoupling protein 3 expression in the rat heart. Diabetes $\mathbf{4 8}$, 430-435.

Kelley DE, He J, Menshikova EV \& Ritov VB (2002) Dysfunction of mitochondria in human skeletal muscle in type 2 diabetes. Diabetes 51, 2944-2950.

Kelley DE \& Mandarino LJ (2000) Fuel selection in human skeletal muscle in insulin resistance: a reexamination. Diabetes 49, 677-683.

Liang P, Hughes V \& Fukagawa NK (1997) Increased prevalence of mitochondrial DNA deletions in skeletal muscle of older individuals with impaired glucose tolerance: possible marker of glycemic stress. Diabetes 46, 920-923.

Listenberger LL, Han X, Lewis SE, Cases S, Farese RV Jr, Ory DS \& Schaffer JE (2003) Triglyceride accumulation protects against fatty acid-induced lipotoxicity. Proceedings of the National Academy of Sciences USA 100, 3077-3082.

Millet L, Vidal H, Andreelli F, Larrouy D, Riou J-P, Ricquier D, Laville M \& Langin D (1997) Increased uncoupling protein-2 and -3 mRNA expression during fasting in obese and lean humans. Journal of Clinical Investigation 100, 2665-2670. 
Pilegaard H, Ordway GA, Saltin B \& Neufer PD (2000) Transcriptional regulation of gene expression in human skeletal muscle during recovery from exercise. American Journal of Physiology 279, E806-E814.

Russell AP, Schrauwen P, Somm E, Gastaldi G, Hesselink MKC, Schaart G, Kornips E, Lo SK, Bufano D, Giacobino J-P, Muzzin P, Ceccon M, Angelini C \& Vergani L (2003a) Decreased fatty acid $\beta$-oxidation in riboflavin-responsive multiple acylCoA dehydrogenase deficient patients is associated with an increase in UCP3. Journal of Clinical Endocrinology and Metabolism 88, 5921-5926.

Russell AP, Wadley G, Hesselink MKC, Schaart G, Lo S, Leger B, Garnham A, Kornips E, Cameron-Smith D, Giacobino JP, Muzzin P, Snow R \& Schrauwen P (2003b) UCP3 protein expression is lower in type I, IIa and IIx muscle fiber types of endurance-trained compared to untrained subjects. Pflugers Archiv European Journal of Physiology 445, 563-569.

Samec S, Seydoux J \& Dulloo AG (1998) Role of UCP homologues in skeletal muscles and brown adipose tissue: mediators of thermogenesis or regulators of lipids as fuel substrate? FASEB Journal 12, 715-724.

Schrauwen P \& Hesselink M (2003) Uncoupling protein 3 and physical activity: the role of uncoupling protein 3 in energy metabolism revisited. Proceedings of the Nutrition Society 62, 635-643.

Schrauwen P, Hesselink MK, Blaak EE, Borghouts LB, Schaart G, Saris WH \& Keizer HA (2001a) Uncoupling protein 3 content is decreased in skeletal muscle of patients with type 2 diabetes. Diabetes 50, 2870-2873.

Schrauwen P, Hesselink MK, Vaartjes I, Kornips E, Saris WH, Giacobino JP \& Russell A (2002a) Effect of acute exercise on uncoupling protein 3 is a fat metabolism-mediated effect. American Journal of Physiology 282, E11-E17.
Schrauwen P, Hinderling V, Hesselink MKC, Schaart G, Kornips E, Saris WHM, Westerterp-Plantega M \& Langhans W (2002b) Etomoxir-induced increase in UCP3 supports a role of uncoupling protein 3 as a mitochondrial fatty acid anion exporter. FASEB Journal 16, 10.1096/fj.1002-0275fje.

Schrauwen P, Hoeks J, Schaart G, Kornips E, Binas B, Vusse GJvd, Bilsen Mv, Luiken JJFP, Coort SLM, Glatz JFC, Saris WHM \& Hesselink MKC (2003) Uncoupling protein 3 as a mitochondrial fatty acid anion exporter. FASEB Journal $\mathbf{1 7}$ 2272-2274.

Schrauwen P, Hoppeler H, Billeter R, Bakker AH \& Pendergast DR (2001b) Fiber type dependent upregulation of human skeletal muscle UCP2 and UCP3 mRNA expression by highfat diet. International Journal of Obesity and Related Metabolic Disorders 25, 449-456.

Schrauwen P, Saris WH \& Hesselink MK (2001c) An alternative function for human uncoupling protein 3 : protection of mitochondria against accumulation of nonesterified fatty acids inside the mitochondrial matrix. FASEB Journal 15, 2497-2502.

Skulachev VP (1999) Anion carriers in fatty acid-mediated physiological uncoupling. Journal of Bioenergetics and Biomembranes 31, 431-445.

Tsuboyama-Kasaoka N, Tsunoda N, Maruyama K, Takahashi M, Kim H, Ikemoto S \& Ezaki O (1998) Up-regulation of uncoupling protein 3 (UCP3) mRNA by exercise training and down-regulation of UCP3 by denervation in skeletal muscles. Biochemical and Biophysical Research Communications 247, 498-503.

Weigle DS, Selfridge LE, Schwartz MW, Seeley RJ, Cummings DE, Havel PJ, Kuijper JL \& BertrandelRio H (1998) Elevated free fatty acids induce uncoupling protein 3 expression in muscle. A potential explanation for the effect of fasting. Diabetes 47, 298-302. 